\title{
Comparative characteristics of white and black ceylon tea based on morphological features of the leaf
}

\author{
Natalya Durnova ${ }^{1 *}$, Margarita Simakova ${ }^{1}$, Daniil Isaev $^{1}$, Alexander Simakov $^{2}$,and Inna Simakova $^{2}$ \\ ${ }^{1}$ Saratov State Medical University named after V.I. Razumovsky, Saratov, Russia \\ ${ }^{2}$ Saratov State Agrarian University named after N.I. Vavilov, Saratov Russia
}

\begin{abstract}
The central question of the research was addressed to comparative characteristics of white and black Ceylon tea based on morphological features of the leaf. The relevance of the study is due significant increasing falsifications of black and white tea on markets in Russia and all around the world. It should be noted that tea is still incomplete and insufficiently studied crop, despite its centuries-old use in human nutrition. Thus, the purpose of this work was to study the microstructure of black and white tea to identify differences in morphological characteristics of the leaf and develop markers for identifying its variety. The objects of study of this work were white and black varieties OP1 teas of Nandana Tea Factory (Sri Lanka). In our research were used research methods includes organoleptic and microscopical methods. According to the results micro- and macroscopic studies of black and white tea samples showed that there are significant morphological differences in the structure of leaves of different varieties, such as the size and density of hairs, the density of stomata, the location of druses and sclereids, and outgrowths of the leaf edge. These signs can serve as reliable markers for identifying the grade of tea.
\end{abstract}

\section{Introduction}

Tea is the oldest and one of the most common drinks in the world, the use of which is related to authentic cuisine and traditions of many nations. For various reasons, many nations consider tea not only as a drink, but also as a first necessity product, a strategic component of the country's food security basket. In accordance with this, tea consumption should be based on modern scientific ideas about the product, knowledge about its benefits, confidence in its quality and safety.

It should be noted that tea is still incomplete and insufficiently studied crop, despite its centuries-old use in human nutrition. And it has been known for more than 5,000 years. For example, today there are contradictions in scientific researches related to the chemical composition of various types of tea and its impact on the human body. Some scientists have pointed out that highquality Chinese green teas have the most valuable mineral composition and the highest protein content in comparison with black and white teas [1]. Results of scientist's works Unachukwu UJ, Ahmed S from Lehman College, Bronx, NY, USA shown that the polyphenols content in green teas is similar to some varieties of white tea; some white teas have comparable amounts of common catechins with some green teas, but less antioxidant capacity. It is assumed to be connected with the less non-catechin antioxidants content than in green teas. [2] While in the research of Santana-Rios G, Orner GA the powerful antimutagenic activity of white tea in comparison with green tea has been revealed. Nine major components found in green tea have also been found in white tea, including epigallocatechin-3-gallate (EGCG) and some other polyphenols. [3] The inconsistency in the conclusions can be probably explained both by a discrepancy in the quality of the studied types of tea to their classification, and by other reasons that affect the chemical composition of the final product and its impact on the human body. In the opinion of the authors of this work, in order to remove contradictions and increase the share of truth, you need to be sure that the tea to be studied belongs to the variety indicated on the packaging in accordance with the international classification of tea varieties. One of the simplest and most accessible tools as markers of authenticity and compliance of tea indicated on the label variety, in addition to the generally accepted organoleptic methods, can be the morphological characteristics of the tea leaf. Unfortunately, there are not enough works in scientific periodicals devoted to the study of morphological features of tea raw materials that identify its grade. There are works devoted to leaf morphology as an identifier of the most important hereditary traits that determine the essential difference between Thea sinensis and Thea assamica varieties. For example, in the book of W. Weixin «Encyclopedia of healing tea» [4] is indicated that when studying the tissues and cells of the tea leaf, it has been found that on the upper and lower sides it consists of single-layer cells of the epidermis, under which, depending on the type of tea bush, there are one, two or three layers of palisade tissue, and under them there are cells of the spongy parenchyma. On the lower surface of the leaf there are stomata and hairs. Most of the hairs are found on young leaves and stems. Scientists Xiaofang Zhu, Yi Zhang 
found that the quality of the aroma of black tea and oolong tea depends on the cuticle wax. However, there is still no comprehensive documentation on the cuticle of the tea leaf [5]. Thus, the purpose of this work was to study the microstructure of black and white tea to identify differences in morphological characteristics of the leaf and develop markers for identifying its variety.

\section{Experimental}

Objects of study. The objects of study of this work were white and black teas of Nandana Tea Factory (Sri Lanka). The share of Ceylon tea in the world production is about $9-10 \%$. In Sri Lanka, different types of tea are produced, only the Assamese variety of the tea plant is cultivated.

The rationale of the research objects is the varying degree of their fermentation during processing and the growth phase of shoots during harvesting. White tea varieties are non-fermented (oxidation up to $12 \%$ of the total amount of tanning substances of the raw material), black tea is fermented - oxidation within the limit of 35$45 \%$ of the total content of tanning substances.

Black tea varieties OP1 in accordance with the international classification. Orange Pekoe, category $1-\mathrm{a}$ variety of high-quality large-leaf tea with an infusion of medium strength. The variety is made from the first and second flushes of fresh leaves which grow on the highest point of the tea tree.

White tea is a drink of elite quality. The composition contains tips and the first upper leaf.

It should be noted that white and black teas belong to baihao teas. Black tea is dominant on the world market, especially prevalent in temperate, cool, humid (wet) climate countries. To get such a tea, they should undergo the leaf three stages of processing: withering, twisting and fermentation. White tea is a very rare, exceptional in quality tea, consisting entirely of tips and first leaves. Its almost colorless infusion combines the advantages of all types of tea: it has a very delicate and light aroma, rich and strong (especially when re-brewing) taste, has a wide range of healing properties.

All five types of tea existing in the world (black, green, yellow, red, white) in all the variety of its types can be made from leaves of the same bush, what indicates how important is the quality of raw materials and method of its processing.

Research methods. The studies were carried out on the basis of the laboratories of the department "Food Technology" of the Federal State Budgetary Educational University of Higher Education "Saratov State Agrarian University named after N.I. Vavilov", and the Department of General Biology, Pharmacognosy and Botany of the Federal State Budgetary Educational Institution of Higher Education "V.I. Razumovsky Saratov State Medical University of the Ministry of Healthcare of the Russian Federation".

The study of raw materials was carried out in accordance with the requirements of GF XIV OFS 1.5.1.0003.15 "Leaves" and OFS 1.5.3.0003.15
"Technique for microscopic and microchemical studies of medicinal plant materials and herbal medicines".

For the analysis of whole leaves, whole leaves or pieces of a leaf plate with an edge and vein, pieces of a leaf from the base and apex, pieces of a petiole (if the leaf has a petiole) were taken.

The raw material was enlightened in the following way: a few slices of the raw materials were placed in a flask or vial, then the sodium hydroxide $5 \%$ solution, diluted with water $(1: 1)$ was added and the raw material was boiled for $2-5$ minutes depending on the thickness and density of objects, avoiding strong softening. Then the contents were poured into the glass, the liquid was drained through 2 to 4 layers of gauze, which closed the glass, and the raw material is washed thoroughly with water, each time pouring water through the same gauze. The beaker content was transferred to a small amount of water in a Petry dish. Particles of the raw material, remaining on the gauze, were washed in the same Petry dish. From the water the pieces were removed with a scalpel or spatula and placed on a glass slide in a drop of the glycerin $33 \%$ solution. Pieces of raw, enlightened and placed on a glass slide, were separated with a scalpel or dissecting needle into two parts, one of them gently turned. A piece of stem was placed on a glass slide. Thin stalks were crushed with a scalpel or reverse end of a dissecting needle to release the epidermis. From the thick stalks the epidermis was removed with the help of dissecting needles or razor, the rough inner parts of the petiole which prevent getting a good micropreparation of the epidermis were also removed. The object was covered with a cover glass, if necessary, lightly fell on the net reverse end of the dissecting needle and gently warmed to remove air bubbles, after cooling, was considered the sheet on both sides and the epidermis of the petiole under a microscope, first at low then at high magnification. At different magnifications, using the macro sheet and screws, the upper and lower epidermis and the deep structure of the sheet located beneath the epidermal (inclusion, vessels, etc.) were explored.

Preparation of cross sections of petioles: slices were made from soaked petioles by clamping pieces of petiole into a bottle stopper (cork plug) or elderberry core. A piece of elderberry or bottle cork was cut in half and a piece of leaf was clamped between the two halves. For the preparation of transverse slices, the surface of the piece should be prepared so that it is strictly perpendicular to the axis of the petiole or vein of the sheet. Prepared sections were placed in a Petry dish with water, from where the sections were removed and viewed under a microscope.

Organoleptic evaluation of white and black tea was carried out in accordance with GOST 32573-2013 Black tea. Specifications (Edition as amended) and GOST 33481-2015 Tea partially fermented. Technical conditions.

\section{Results and discussion}

At the first stage of work, an organoleptic evaluation of tea raw materials was carried out. 
A photograph of black tea is shown in Fig. 1. Leaves are whole and well twisted, homogeneous. Kidneys do not occur. The sizes of the leaves are from 0.5 to $2.0 \mathrm{~cm}$ in length. Sometimes there are pieces of the stem, they are more straight, stiff, black, about $1.0-1.5 \mathrm{~cm}$ in length. Tea infusion is homogeneous, brown-red. It has a wonderful aroma by brewing and tart flavour. In appearance, black tea, grade OP1, meets the requirements of GOST 32573-2013 and the international classification of tea varieties.

The dried raw materials of white tea (Fig. 2) are solid leaves of silver-green colour with a petiole, with copious pubescence on the underside of the leaf.

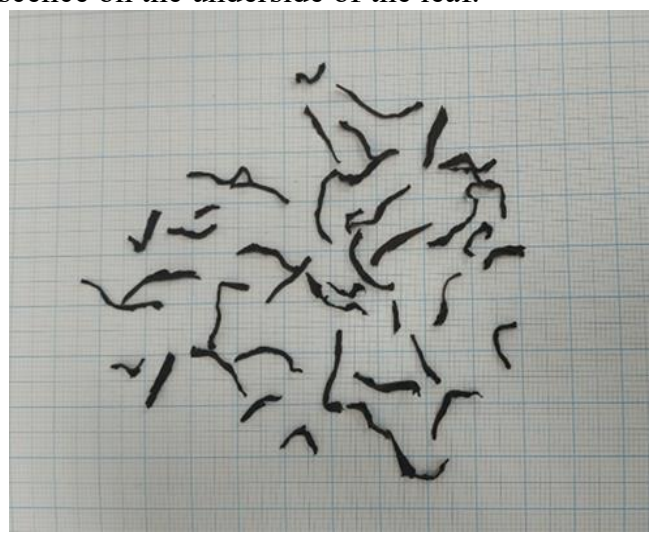

Fig. 1. Photo of raw black tea.

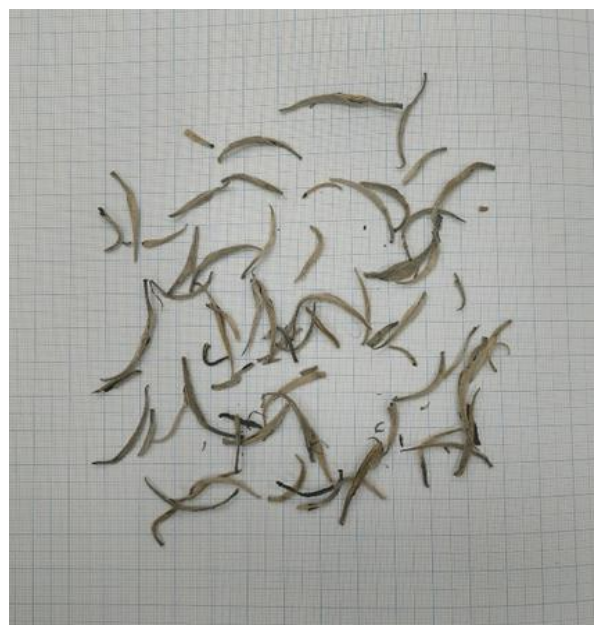

Fig. 2. Photo of raw white tea.

A leaf blade from the edges is turned inward through the ventral side. The sheet's size is $1.2-3.0 \mathrm{~cm}$ in length and up to $0.5 \mathrm{~cm}$ in width has a crescent-shaped bend to the dorsal side. The main vein is visible with the naked eye on the underside of the leaf. Fragmented parts of leaves are occasionally found. Inside some leaves, tips are found that are morphologically similar to the rest of the leaves, but smaller and folded into a tube. Tea infusion is clear, taste and smell are floral, delicate, well perceptible. Organoleptic characteristics of white tea brand Nandana tea factory (Sri Lanka) corresponds to GOST 33481-2015 Tea is partially fermented. Specifications and international classification of tea varieties and refers to white types of tea.

After brewing the dried raw black tea (Fig. 3), the leaves unfold, parts of leaf blades and central veins become well distinguishable. Unfolded raw materials are usually pieces of stems, less often the central vein without a leaf blade. The leaf blade is heterogeneous in color, from dark to light green. Dimensions usually do not exceed $1 \times 1 \mathrm{~cm}$. The edge of the leaf remaining on some parts of the leaf blades is smooth, slightly serrated. The top of the leaf is sharp; the base is wedge-shape. The leaf blade is thin.

The raw material of white tea does not change shape after brewing (Fig. 4), but the color of the leaves changes from silver-green to dark green, almost black. Tips are clearly visible. The leaf is quite thick, brittle, when trying to take a sample for a micropreparation, the upper and lower epidermis peeled off easily, and the sheet itself broke.

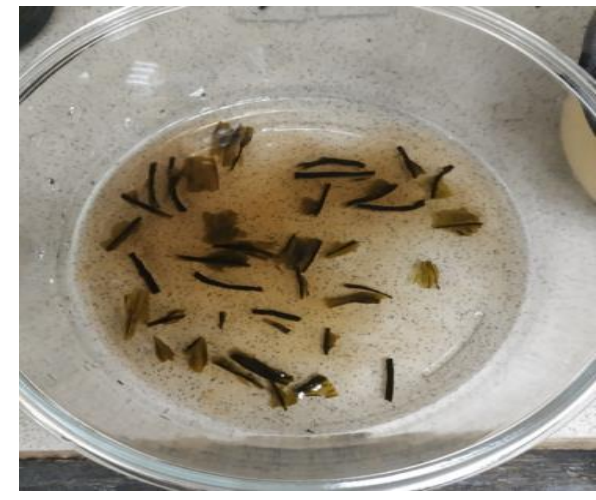

Fig. 3. Raw black tea after brewing.

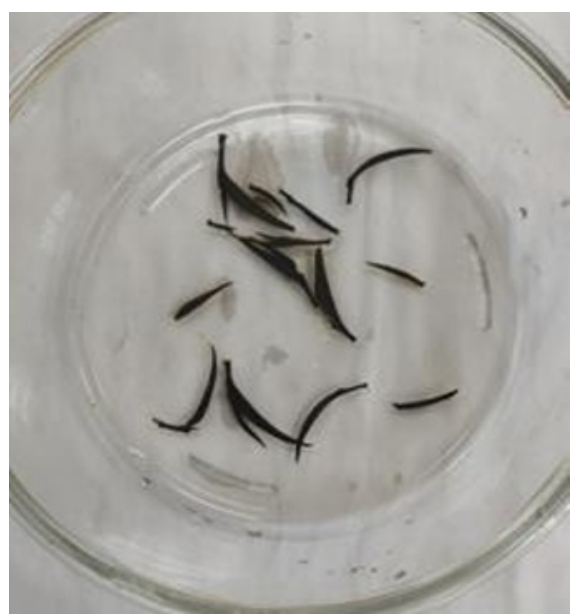

Fig. 4. Raw white tea after brewing.

Microphotographs of tea preparations are shown in Fig. 5 and 6.

Fig. 5 shows a micrograph of a leaf of black tea. One can see a large number of stomata per surface unit, rare hairs, clusters of druses, small veins that are not in focus, can be also seen.

Fig. 6 shows a micrograph of white tea, where a large number of hairs and outgrowths of the leaf blade, which are not visible to the naked eye, can be seen at a glance.

On the lower epidermis of white tea (Fig. 7-8), such a high density of hairs is observed that they completely overlap the overview of the underlying structures. The places of attachment of hairs to the epidermis are visible.

The epidermal cells with some cellular structures and the attachment sites of hairs are clearly visible. It can be 
seen that between adjacent hairs from 1 to 3 epidermal cells are located.

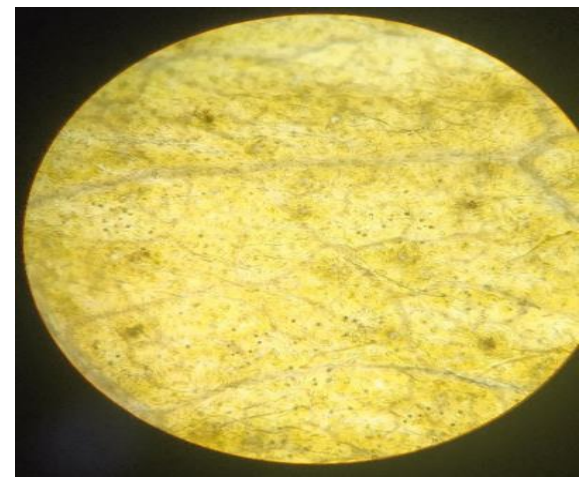

Fig. 5. A leaf blade of black tea. Magnification $10 \times 10$.

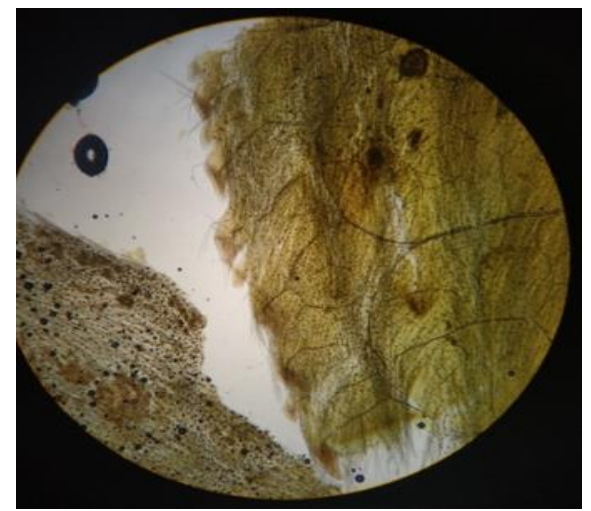

Fig. 6. Leaf plate of white tea. Magnification $10 \times 4$.

Black tea hairs are rarer and shorter than white tea hairs (Fig. 9). Hairs are unicellular.

Figures 10-11 clearly show the stomata as well as the cuticle folds. Presumably these are stomata of paracytic type. The guard cells and their thickened inner wall are visible. Sometimes stomata are found whose size exceeds the size of the surrounding stomata.

Stomata on white tea (Fig. 12) are extremely difficult to find and examine because they are rare, and hairs hinder the review. Only two guard cells are poorly distinguishable.

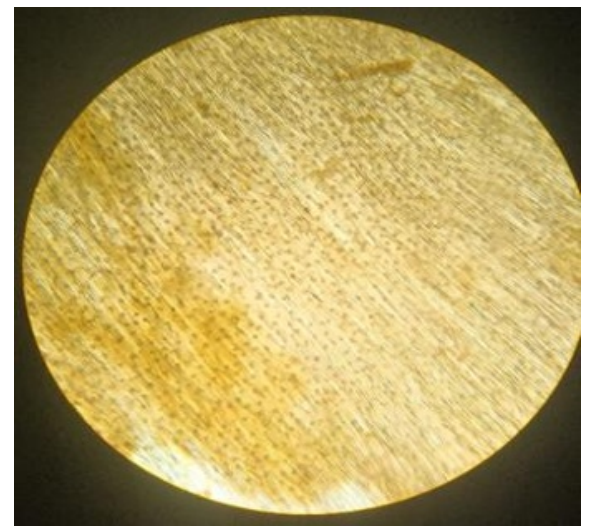

Fig. 7. Hairs of white tea. Magnification 10×10.

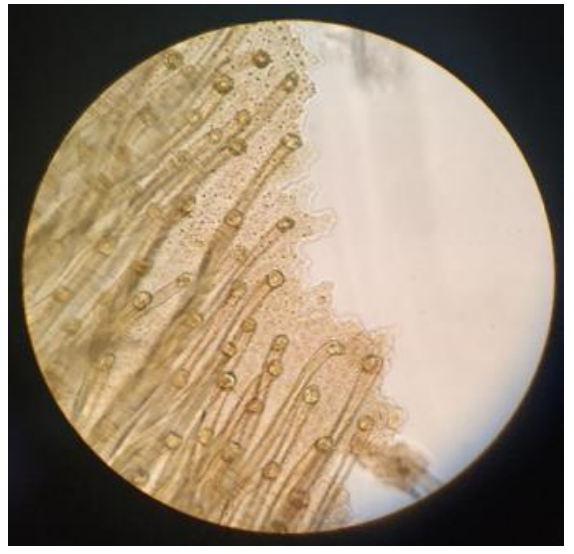

Fig. 8. Hairs of white tea. Magnification $10 \times 40$.

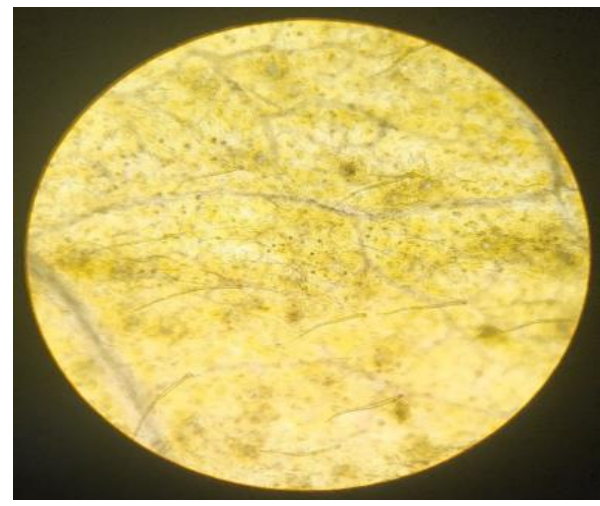

Fig. 9. Hairs of black tea. Magnification $10 \times 10$.

In conducting bundles of black tea (Fig. 13-14), spiral thickenings of the vessel walls are clearly visible. Thicker vessels are located in the center of the conducting bundle; to the periphery, the diameter of the vessels decreases. In some areas, the conducting bundles are surrounded by several (2-3) even rows of druses.

In conducting bundles of white tea (Fig. 15), spiral thickenings of the vessel wall are clearly visible. Thicker vessels are located in the center of the conducting bundle; to the periphery, the diameter of the vessels decreases.

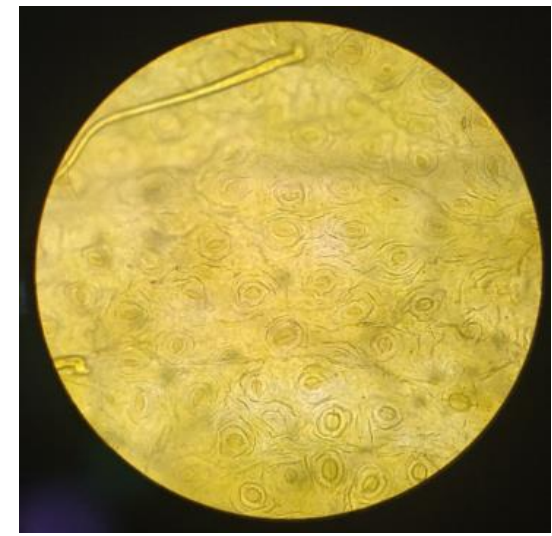

Fig. 10. Stomata of black tea. Magnification $10 \times 40$. 


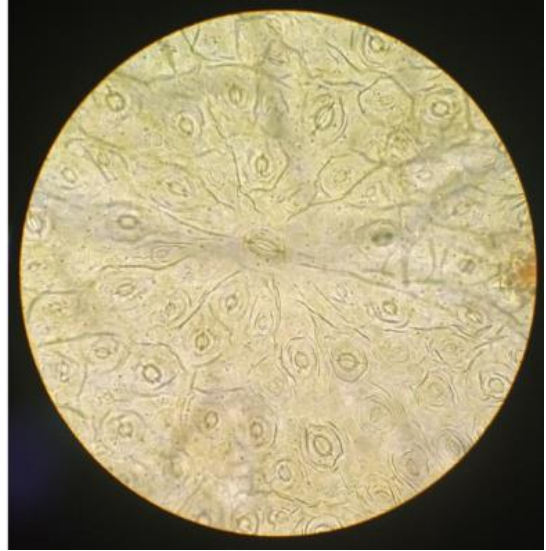

Fig. 11. Stomata of white tea. Magnification $10 \times 40$.

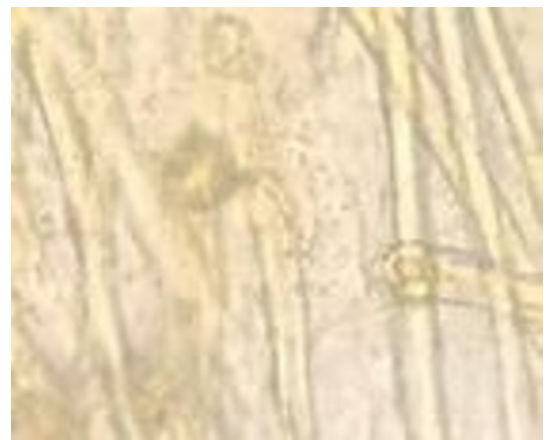

Fig. 12 Stomata of white tea. Magnification $10 \times 40$.

Reduced structures representing the tops of the serrated edges of the leaf are visible in some areas of the leaf blade of black tea (Fig. 16-17).

It can be seen that, as in white tea, in black tea, the conducting bundles are approaching to these outgrowths. The outgrowths apparently do not play a significant role at this stage of the leaf development. Fig. 17 shows a dead, partially damaged, lignified, but preserved almost entirely on its original place outgrowth.

Along the entire edge of the white tea leaf in the middle of the leaf blade, such outgrowths with vessels, which are approaching to them, are observed (Fig. 1819). Morphologically, these outgrowths are similar to the top of a leaf plate (Fig. 20). It is visibly that the outgrowths of the leaf edge can be heterogeneous in size and shape, but have the same general structure.

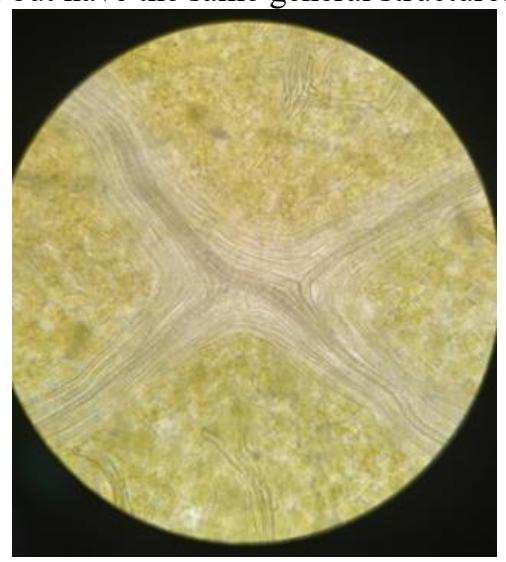

Fig. 13. Conducting bundles of black tea. Magnification $10 \times 40$.

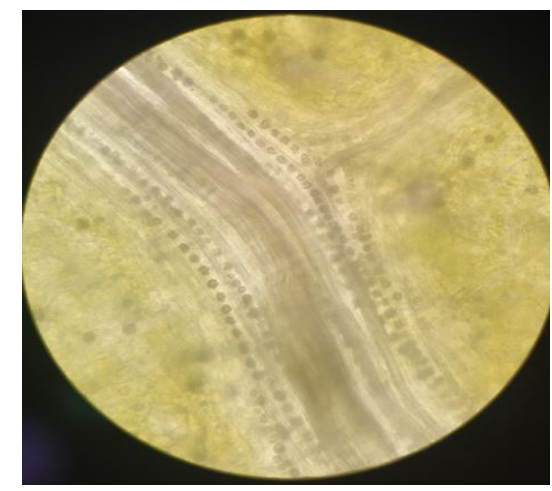

Fig. 14. Conducting bundles of black tea. Magnification $10 \times 100$.

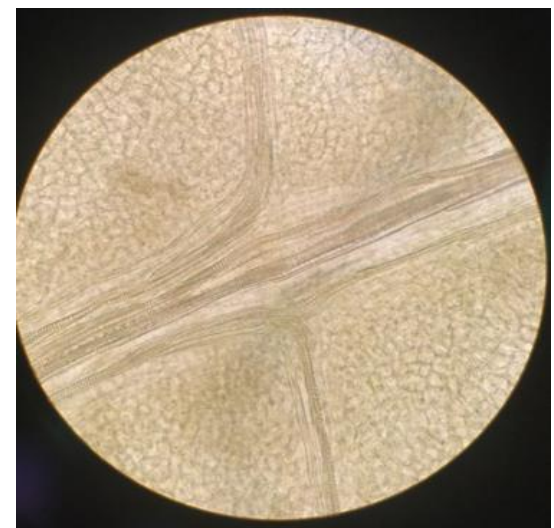

Fig. 15. Conducting bundles of white tea. Magnification $10 \times 40$.

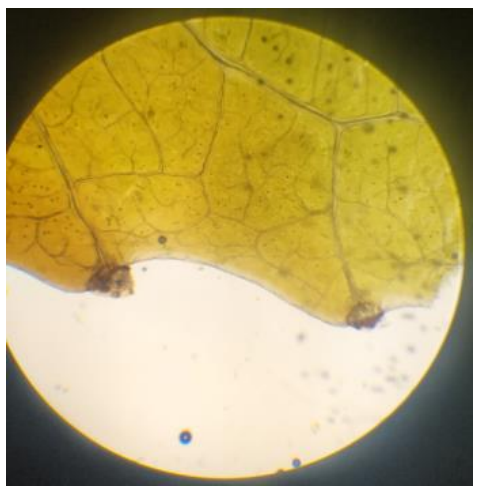

Fig. 16. Outgrowths of the leaf edge of black tea. Magnification $10 \times 40$.

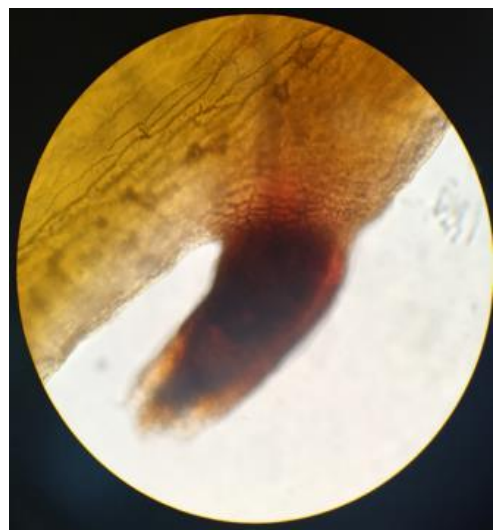

Fig. 17. Outgrowths of the leaf edge of black tea. Magnification $10 \times 40$. 


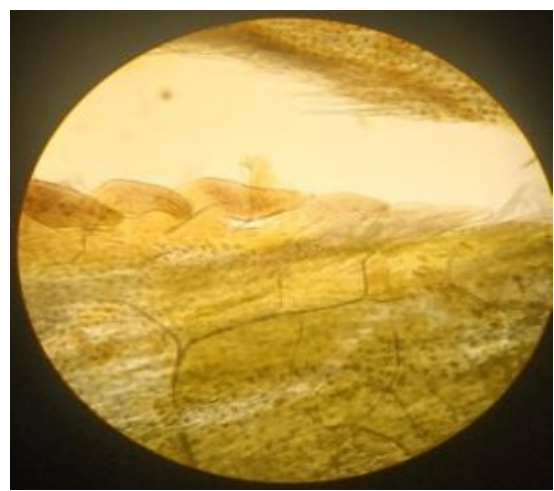

Fig. 18. Outgrowths of the edge of a leaf of white tea. Magnification $10 \times 10$.

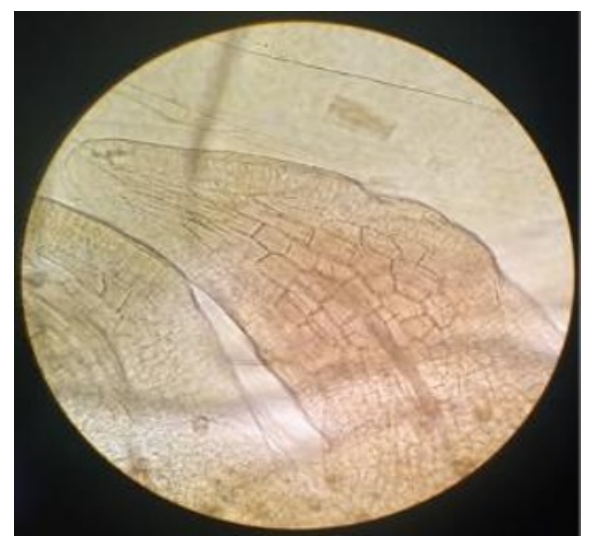

Fig. 19. Outgrowths of the edge of a leaf of white tea. Magnification $10 \times 100$.

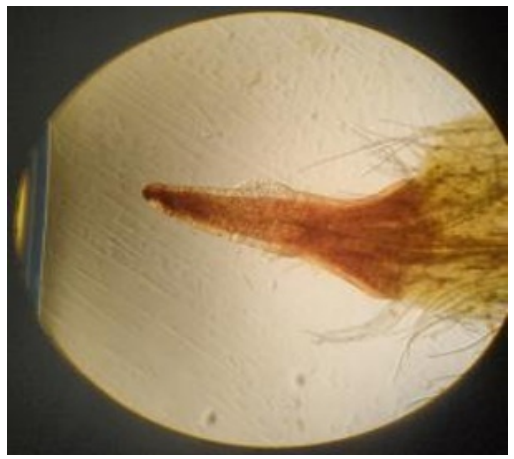

Fig. 20. Outgrowths of the edge of a leaf of white tea. Magnification $10 \times 10$

Astrosclereids in the leaves of black tea (Fig. 21) are very common anywhere in the leaf blade and have the most diverse form. Druses in the leaves of black tea are found everywhere. Often they are concentrated in even rows along the periphery of the conducting bundles (Fig. 14).

In a section of the central vein near the base of a leaf of black tea (Fig. 22), two very large, almost rectangular, sclereids are located at both ends of the conducting bundle on the border with the leaf blade.

Sclereids in white tea leaves (Fig. 23) are found near the top of the leaf; they were not found in the rest of the leaf blade. The edge of the leaf near the apex is whole. Druses are found near the apex and are concentrated at the edge of the leaf. Druses, like sclereids, were not found in other parts of the white tea leaf.
The upper epidermis of the leaf of black tea is represented by highly sinuous cells (Fig. 24).

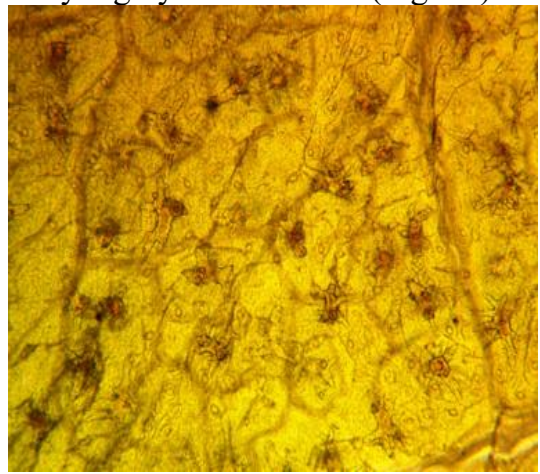

Fig. 21. Sclereids in a leaf plate of black tea. Magnification $10 \times 10$.

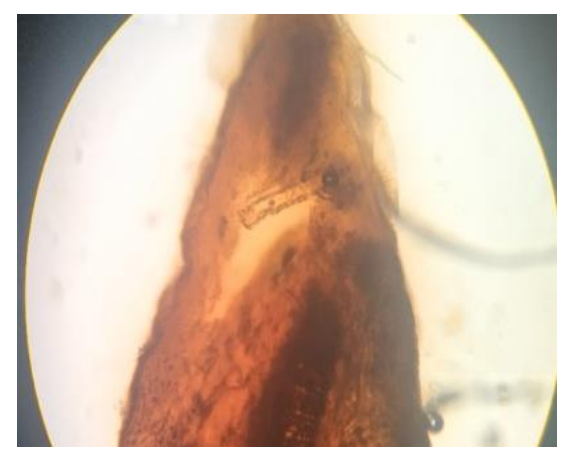

Fig. 22. Large sclereid on a slice of black tea leaf. Magnification $10 \times 4$.

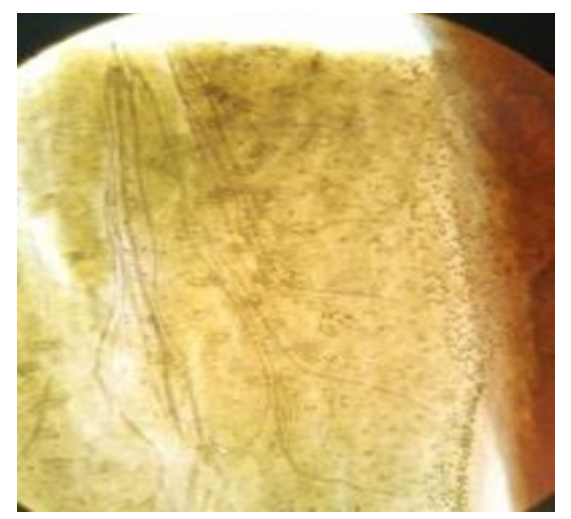

Fig. 23. Druses and sclereids of a leaf of white tea. Magnification $10 \times 4$.

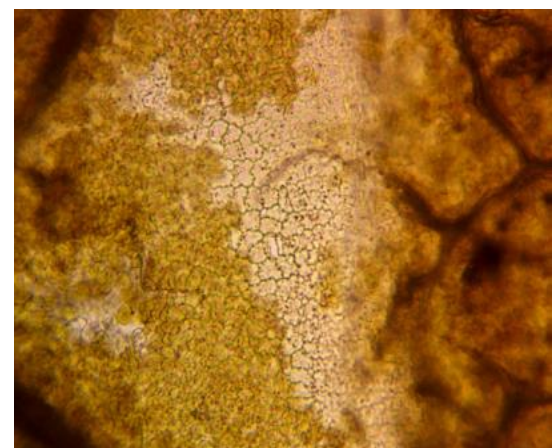

Fig. 24. The upper epidermis of a leaf of black tea Magnification $10 \times 40$. 


\section{Conclusion}

According to the results of a macroscopic research, the raw material of white tea is a flush of 1-2 leaves and a leaf bud of silver-green color, curled up, not too dry, of medium size. Black tea - black, dry, angular, wrinkled parts of the leaf blade and pieces of the stem.

Microscopic examination of white tea showed that the density of hairs on its leaves is extremely high, the hairs are unicellular, long. Stomata are rare; they are difficult to detect and examine. Root buds of denticles are found along the entire edge of the leaf. Sclereids and druses are found only at the top of the leaf blade.

The density of hairs on the leaves of black tea is much lower, and the hairs themselves are shorter. The density of stomata, on the contrary, is much higher. Around the conductive beams are several even rows of druses. The ends of the cloves on the edge of the leaf of black tea are lignified and fall. Sclereids and druses are distributed throughout the leaf blade.

Thus, micro- and macroscopic studies of black and white tea samples showed that there are significant morphological differences in the structure of leaves of different varieties, such as the size and density of hairs, the density of stomata, the location of druses and sclereids, and outgrowths of the leaf edge. These signs can serve as reliable markers for identifying the grade of tea.

\section{Acknowledgment}

The authors thank Nandana Tea Factory and personally Mr. Gunasoma Wanigasekara for providing tea samples for research.

\section{References}

1. M. Czernicka, G. Zaguła, M. Bajcar, B. Saletnik, and C. Puchalski, Rocz Panstw Zakl Hig., 68(3), 237-245 (2017)

2. U.J. Unachukwu, S. Ahmed, A. Kavalier, J.T. Lyles, and E.J. Kennelly, J.Food Sci., 75(6), 541-548, (2010)

3. G. Santana-Rios and G.A. Orner, "Potent antimutagenic activity of white tea in comparison with green tea in the Salmonella assay”, 495(1-2), 61-74, (2001)

4. Electronic document: access mode https://med.wikireading.ru/76027

5. X. Zhu, Y. Zhang, Z. Du, X. Chen, X. Zhou, X. Kong, W. Sun, Z. Chen, C. Chen, and M. Chen, Reports, 8(1), 14944 (2018) 